\title{
THE SCHÜTZENBERGER CATEGORY OF A SEMIGROUP
}

\author{
ALFREDO COSTA AND BENJAMIN STEINBERG
}

\begin{abstract}
In this paper we introduce the Schützenberger category $\mathbb{D}(S)$ of a semigroup $S$. It stands in relation to the Karoubi envelope (or Cauchy completion) of $S$ in the same way that Schützenberger groups do to maximal subgroups and that the local divisors of Diekert do to the local monoids $e S e$ of $S$ with $e \in E(S)$. In particular, the objects of $\mathbb{D}(S)$ are the elements of $S$, two objects of $\mathbb{D}(S)$ are isomorphic if and only if the corresponding semigroup elements are $\mathscr{D}$-equivalent, the endomorphism monoid at $s$ is the local divisor in the sense of Diekert and the automorphism group at $s$ is the Schützenberger group of the $\mathscr{H}$-class of $S$. This makes transparent many well-known properties of Green's relations.

The paper also establishes a number of technical results about the Karoubi envelope and Schützenberger category that were used by the authors in a companion paper on syntactic invariants of flow equivalence of symbolic dynamical systems.
\end{abstract}

\section{INTRODUCTION}

Small categories have played an important role in semigroup theory for a long time, see for example [13, 15, 19, 22]. One of the first categories considered in this context was the Karoubi envelope $\mathbb{K}(S)$ of a semigroup $S$; it plays a fundamental role in Tilson's Delay Theorem [22] (see [10] and 20] for its predecessors). Informally, the objects of $\mathbb{K}(S)$ are idempotents of $S$ and $\mathbb{K}(S)\left(e, e^{\prime}\right)=e^{\prime} S e$. Composition is induced by the multiplication in $S$. It is easy to check that isomorphism in $\mathbb{K}(S)$ corresponds to $\mathscr{D}$-equivalence, the endomorphism monoids are the so-called local monoids $e S e$ and the automorphism groups are the maximal subgroups.

Date: September 17, 2018.

2010 Mathematics Subject Classification. 18B99, 20M10, 20M50.

The first author was supported by the Centro de Matemática da Universidade de Coimbra (CMUC), funded by the European Regional Development Fund through the program COMPETE and by the Portuguese Government through the Fundação para a Ciência e a Tecnologia (FCT) under the project PEst-C/MAT/UI0324/2013. He was also supported by the FCT post-doctoral grant SFRH/BPD/46415/2008 and by the FCT project PTDC/MAT/65481/2006, within the framework of European programmes COMPETE and FEDER. The second author was partially supported by a grant from the Simons Foundation (\#245268 to Benjamin Steinberg) and the Binational Science Foundation of Israel and the US (\#2012080 to Benjamin Steinberg). Some of this work was performed while the second author was at the School of Mathematics and Statistics, Carleton University, Ottawa, Canada under the auspices of an NSERC grant. 
Schützenberger, long ago, associated a group, now called the Schützenberger group [4], to each $\mathscr{H}$-class of a semigroup which coincides with the maximal subgroup when considering the $\mathscr{H}$-class of an idempotent. Recently, Diekert and his coauthors have associated a monoid to each element $s$ of a semigroup $S$, called a local divisor [7 9], which in the case that $s=e$ is an idempotent reduces to $e S e$ and whose group of units is the Schützenberger group of the $\mathscr{H}$-class of $s$.

Here we introduce a small category $\mathbb{D}(S)$, which we call the Schützenberger category of $S$, whose objects are elements of $S$. The endomorphism monoid at an object $s$ will be Diekert's local divisor associated to $s$ and the automorphism group will be the Schützenberger group of the $\mathscr{H}$-class of $s$. Two objects of $\mathbb{D}(S)$ are isomorphic if and only if they are $\mathscr{D}$-equivalent (whence the notation). The Karoubi envelope is isomorphic to the full subcategory of $\mathbb{D}(S)$ on the idempotents. The construction satisfies $\mathbb{D}\left(S^{o p}\right)=\mathbb{D}(S)^{o p}$ and so we obtain a transparent proof that the left and right Schützenberger groups of an $\mathscr{H}$-class are isomorphic and depend only on the $\mathscr{D}$-class up to isomorphism.

This paper is intended as a companion to [6. In that paper, it is shown that if $\mathscr{X}$ is a symbolic dynamical system and $S(\mathscr{X})$ is the syntactic semigroup of the language of blocks of $\mathscr{X}$, then $\mathbb{K}(S(\mathscr{X})$ ) (up to natural equivalence) is a flow equivalence invariant of $\mathscr{X}$. Flow equivalence is a highly studied coarsening of topological conjugacy. See [14] for details on symbolic dynamics. In [6], we needed some purely algebraic properties of $\mathbb{K}(S)$ and $\mathbb{D}(S)$ whose proofs were relegated to this paper, as they had nothing to do with dynamics.

The paper is organized as follows. After a brief section on categorical preliminaries, we introduce the Karoubi envelope and Schützenberger category of a semigroup, first as a concrete category, and then abstractly. The following section discusses the connection between actions of semigroups and actions of their associated categories. We also prove the technical result that if $S$ and $T$ have local units, then $\mathbb{K}(S)$ equivalent to $\mathbb{K}(T)$ implies that $\mathbb{D}(S)$ is equivalent to $\mathbb{D}(T)$. In the final section, we consider several invariants of an action of a semigroup on a set that are detected by the Karoubi envelope. These invariants are used in [6] to obtain flow equivalence invariants of symbolic dynamical systems.

\section{Categorical Preliminaries}

The reader is referred to [1,3, 16] for basic notions from category theory. A category $C$ is small if its objects and arrows form a set. We often write $C\left(c, c^{\prime}\right)$ for the set of morphisms from $c$ to $c^{\prime}$. Note that we compose arrows as traditionally done by category theorists: if $f: c \rightarrow c^{\prime}$ and $g: c^{\prime} \rightarrow c^{\prime \prime}$, then their composition is $g f: c \rightarrow c^{\prime \prime}$. The identity at an object $c$ is usually denoted $1_{c}$. 
A natural transformation $\eta: F \Rightarrow G$ between functors $F, G: C \rightarrow D$ is an assignment of an arrow $\eta_{c}: F(c) \rightarrow G(c)$ for each object $c$ of $C$ such that, for any arrow $f: c \rightarrow c^{\prime}$ of $C$, the diagram

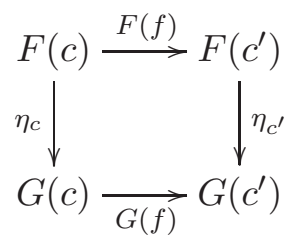

commutes. There is then a category $D^{C}$ with objects functors from $C$ to $D$ and arrows natural transformations between them. Two functors $F, G: C \rightarrow$ $D$ are isomorphic, written $F \cong G$, if they are isomorphic in $D^{C}$.

We recall that two categories $C$ and $D$ are equivalent if there are functors $F: C \rightarrow D$ and $G: D \rightarrow C$ such that $F G \cong 1_{D}$ and $G F \cong 1_{C}$. A functor $F: C \rightarrow D$ between small categories is an equivalence (i.e., there exists such a $G$, called a quasi-inverse) if and only if it is fully faithful and essentially surjective. Fully faithful means bijective on hom-sets (i.e., $F: C\left(c, c^{\prime}\right) \rightarrow D\left(F(c), F\left(c^{\prime}\right)\right)$ is a bijection for all $\left.c, c^{\prime}\right)$, whereas essentially surjective means that every object of $D$ is isomorphic to an object in the image of $F$. The former is in accordance with the usual terminology for functors which are injective on hom-sets (the faithful functors) and for those which are surjective on hom-sets (the full functors).

\section{The Karoubi envelope and Schützenberger Category of A SEMIGROUP}

3.1. Inner morphisms of principal right ideals. Let $S$ be a semigroup. A right $S$-set is a set $X$ equipped with a right action by $S$. Key examples include principal right ideals $s S^{1}$ (where $S^{1}$ is the result of adjoining an external identity to $S$ ). Note that if $s$ is regular, then $s S^{1}=s S$ and we often write the latter. If $S$ is a monoid (or more generally has local units, see below) then the projective indecomposable $S$-sets are precisely those isomorphic to one of the form $e S^{1}=e S$ for some idempotent $e \in S$.

A morphism of right $S$-sets is a mapping $f: X \rightarrow Y$ such that $f(x s)=$ $f(x) s$ for all $x \in X$ and $s \in S$. We say that a morphism $f: s S^{1} \rightarrow t S^{1}$ of principal right ideals is inner if there exists $u \in S^{1}$ such that $f(x)=u x$ for all $x \in s S^{1}$. We do not claim that $u$ is unique. By an inner isomorphism, we mean one whose inverse is also inner. Let us say that $s S^{1}$ and $t S^{1}$ are inner isomorphic if there is an inner isomorphism between them. We write $\operatorname{hom}\left(s S^{1}, t S^{1}\right)$ for the set of inner morphisms $s S^{1} \rightarrow t S^{1}$.

We record here some basic facts about morphisms.

Proposition 3.1. Let $S$ be a semigroup.

(1) If $f, g: s S^{1} \rightarrow t S^{1}$ are morphisms, then $f=g$ if and only if $f(s)=$ $g(s)$. 
(2) Left multiplication by an element $u \in S^{1}$ induces an inner morphism $s S^{1} \rightarrow t S^{1}$ if and only if $u \in\left(t S^{1}\right) s^{-1}=\left\{v \in S^{1} \mid v s \in t S^{1}\right\}$, if and only if us $\in t S^{1}$.

(3) Two elements $u, v$ of $\left(t S^{1}\right) s^{-1}$ induce the same inner morphism if and only if $u s=v s$.

(4) The mapping $f \mapsto f(s)$ gives a bijection $\operatorname{hom}\left(s S^{1}, t S^{1}\right) \rightarrow t S^{1} \cap S^{1} s$.

(5) If e, $f$ are idempotents, then $\operatorname{hom}(e S, f S)$ is in bijection with $f S e=$ $f S \cap S e$ via $g \mapsto g(e)$.

(6) If $s, t$ are regular, then every morphism $s S \rightarrow t S$ is inner.

(7) The composition of inner morphisms is inner and hence we can consider the category of principal right ideals of $S$ with inner morphisms. More precisely, if $v$ induces an inner morphism $s S^{1} \rightarrow t S^{1}$ and $w$ induces an inner morphism $t S^{1} \rightarrow u S^{1}$, then their composition $s S^{1} \rightarrow u S^{1}$ is the inner morphism induced by wv.

(8) An inner morphism $f: s S^{1} \rightarrow t S^{1}$ corresponding to $w=f(s) \in$ $t S^{1} \cap S^{1} s$ is an inner isomorphism if and only if $t \mathscr{R} w \mathscr{L} s$.

(9) $s \mathscr{D} t$ if and only if $s S^{1}$ and $t S^{1}$ are inner isomorphic.

(10) The action of the group of inner automorphisms of $s S^{1}$ restricts to a free and transitive action on the $\mathscr{H}$-class $H_{s}$. Thus we can identify the group of inner automorphisms of $s S^{1}$ with the left Schützenberger group of $H_{s}$.

Proof. If $f(s)=g(s)$, then $f(s x)=f(s) x=g(s) x=g(s x)$ for all $x \in S^{1}$ establishing the first item. If $x \mapsto u x$ is an inner morphism $s S^{1} \rightarrow t S^{1}$, then in particular $u s \in t S^{1}$. Conversely, if $u s \in t S^{1}$, then obviously usx $\in t S^{1}$ for all $x \in S^{1}$. This establishes the second item. The third item is immediate from the first. If $f \in \operatorname{hom}\left(s S^{1}, t S^{1}\right)$ with $f(x)=u x$, then $f(s)=u s \in$ $t S^{1} \cap S^{1} s$ and the map $f \mapsto f(s)$ is injective by the first item. If $v \in t S^{1} \cap S^{1} s$ and $v=u s$ with $u \in S^{1}$, then by the second item, $u$ induces an inner morphism $f: s S^{1} \rightarrow t S^{1}$ with $f(s)=u s=v$. This proves the fourth item. The fifth item is a special case of the fourth item.

Suppose that $s s^{\prime} s=s$ and $t t^{\prime} t=t$. Let $f: s S \rightarrow t S$ be a morphism and put $u=f\left(s s^{\prime}\right)$. Then $u s=f\left(s s^{\prime}\right) s=f\left(s s^{\prime} s\right)=f(s) \in t S^{1}$. Thus $u$ induces an inner morphism which agrees with $f$ on $s$ and hence on $s S^{1}$ by the first item. This proves the sixth item. The seventh item is trivial.

For the eighth item, suppose first that $f$ has inner inverse $g$. Say that $f(x)=u x$ and $g(y)=v y$. Then $f(s)=u s$ and $s=g f(s)=v f(s)$. Thus $s \mathscr{L} f(s)$. Clearly, $f(s) \in t S^{1}$. Since $f$ is surjective, there exists $z \in S^{1}$ with $t=f(s z)=f(s) z$. Thus $f(s) \mathscr{R} t$. Conversely, suppose that $f$ is an inner morphism with $t \mathscr{R} f(s) \mathscr{L} s$. Say $f(x)=u x$. Then $f(s)=u s$ and so we have $s=y u s$ for some $y \in S^{1}$. Then since yus $\in s S^{1}$, left multiplication by $y$ induces an inner morphism $g: t S^{1}=u s S^{1} \rightarrow s S^{1}$. We claim that $f, g$ are inverses. Indeed, $g f(s)=g(u s)=y u s=s$ and so $g f$ is the identity by the first item. Similarly, $f g(u s)=f(y u s)=f(s)=u s$. We again conclude from the first item that $f g$ is the identity. 
To prove the ninth item, we directly observe from the eight item that if $s S^{1}$ and $t S^{1}$ are inner isomorphic, then $s \mathscr{D} t$. Conversely, if $s \mathscr{L} y \mathscr{R} t$, then $y=u s$ for some $u \in S^{1}$. Thus $f: s S^{1} \rightarrow t S^{1}$ given by $f(x)=u x$ is an inner morphism with $f(s)=u s=y$ and $s \mathscr{L} y \mathscr{R} t$. Thus $f$ is an inner isomorphism by the previous item.

For the final item, let $G$ be the group of inner automorphisms of $s S^{1}$. If $g \in G$, then $s \mathscr{H} g(s)$ by item (8). As the $\mathscr{H}$-class $H_{s}$ of $s$ is contained in the set of generators of $s S^{1}$ as a right ideal, it follows that $H_{s}$ is $G$-invariant. The action on $H_{s}$ is free by (1). If $w \in H_{s}$, then the inner morphism $f: s S^{1} \rightarrow s S^{1}$ with $f(s)=w$ is an inner automorphism by (8) and so $G$ is transitive on $H_{s}$. Recall that the left Schützenberger group $G\left(H_{s}\right)$ consists of all mappings $f: H_{s} \rightarrow H_{s}$ such that, for some $u \in S^{1}$, one has $f(x)=u x$ for all $x \in H_{s}$. But then $u s \in H_{s}$ implies that $f$ extends uniquely to an inner automorphism of $s S^{1}$ by (1) and (8). We conclude that $\left.f \mapsto f\right|_{H_{s}}$ gives an isomorphism of $G$ with $G\left(H_{s}\right)$.

Dual notions and results hold for principal left ideals.

Proposition 3.1 provides a new proof that $s \mathscr{D} t$ implies that the left Schützenberger groups of $H_{s}$ and $H_{t}$ are isomorphic. The classical proof makes use of right Schützenberger groups. We will soon have a new proof that the right and left Schützenberger groups are isomophic.

3.2. The Karoubi envelope of a semigroup. We now present an algebraic model of the category of idempotent-generated principal right ideals of $S$ and their morphisms (all of which are inner by Proposition 3.1). The construction is quite well known to category theorists in a more general context (cf. [1]). In semigroup theory, this category was first considered by Tilson [22] in connection with the Delay Theorem; see also [18, Chapter 4] where some basic properties are exposited.

Let $S$ be a semigroup. The Karoubi envelope $\mathbb{K}(S)$ (also known as Cauchy completion or idempotent splitting) of a semigroup $S$ is a small category whose object set is the set $E(S)$ of idempotents of $S$. A morphism from $f$ to $e$ is a triple $(e, s, f)$ where $s \in e S f$. Such a morphism will be represented by an arrow $e \longleftarrow f$ for reasons to be explained in the next paragraph. Composition of morphisms is given by

$$
(e, s, f)(f, t, g)=(e, s t, g) .
$$

The identity at $e$ is $(e, e, e)$

In Tilson's work, and in most subsequent work in Semigroup Theory, a triple $(e, s, f)$ such that $s \in e S f$ is viewed as a morphism with domain $e$ and codomain $f$, and the composition of morphisms is taken from left to right, which is the opposite of the convention usually followed in Category Theory. Accordingly to this alternate convention, we still have $(e, s, f)(f, t, g)=(e, s t, g)$. In this paper, we adopt the Category Theory convention for composition. However, we do not want to deviate from the 
Semigroup Theory notation, and that is why we represent graphically a morphism $(e, s, f)$ as an arrow $e \longleftarrow f$ with the source on the right. This same convention will also be used later for the Schützenberger category of a semigroup. All other categories will be treated as usual with arrows drawn from left to right.

If $e$ is an idempotent of the semigroup $S$, then $e S e$ is a a monoid with identity $e$, which is called the local monoid of $S$ at $e$. The local monoid of a category $C$ at an object $c$ is the endomorphism monoid of $c$ in $C$. The local monoids of $S$ correspond to the local monoids of $\mathbb{K}(S)$, more precisely, eSe and $\mathbb{K}(S)(e, e)$ are isomorphic for every $e \in E(S)$.

The following result is well known.

Theorem 3.2. The Karoubi envelope $\mathbb{K}(S)$ of a semigroup $S$ is equivalent to the category of idempotent-generated principal right ideals of $S$ and their $S$-set morphisms via the functor given on objects by $e \mapsto e S$.

Proof. Let $C$ be the latter category and define a functor $F: \mathbb{K}(S) \rightarrow C$ on objects by $e \mapsto e S$ and on arrows by $(e, s, f) \mapsto(x \mapsto s x)$. Note that $F$ is surjective on objects and sends $\mathbb{K}(S)(f, e)$ bijectively to hom $(f S, e S)$ by Proposition 3.1. It remains to show that $F$ is a functor. Note that $F(e, e, e)$ is the map $e S \rightarrow e S$ sending $x \rightarrow e x$, but $e x=x$ for all $x \in e S$ and so $F(e, e, e)=1_{e S}$. Next we compute $F(e, s, f) F(f, t, g)(x)=s(t x)=(s t) x=$ $F(e, s t, g)(x)$. This completes the proof that $F$ is an equivalence.

Note that $\mathbb{K}(S)^{o p}=\mathbb{K}\left(S^{o p}\right)$ and hence the category of idempotent-generated principal left ideals is equivalent to the dual of the category of idempotentgenerated principal right ideals.

It follows from Proposition 3.1 that two objects $e, f$ of $\mathbb{K}(S)$ are isomorphic if and only if they are $\mathscr{D}$-equivalent.

An element $s$ of a semigroup $S$ has local units $e$ and $f$, where $e$ and $f$ are idempotents of $S$, if $s=e s f$. The set $L U(S)=E(S) S E(S)$ of elements of $S$ with local units is a subsemigroup of $S$. If $L U(S)=S$, then we say that $S$ has local units. In general, $L U(S)$ is the largest subsemigroup of $S$ which has local units (it may be empty). Clearly $\mathbb{K}(S)=\mathbb{K}(L U(S))$ and so the Karoubi envelope does not distinguish between $S$ and $L U(S)$.

Talwar defined in [21] a notion of Morita equivalence of semigroups with local units in terms of equivalence of certain categories of actions. It was shown in [11,12] that semigroups $S$ and $T$ with local units are Morita equivalent if and only if $\mathbb{K}(S)$ and $\mathbb{K}(T)$ are equivalent categories.

3.3. The Schützenberger category of a semigroup. Next, we define an algebraic model of the category of principal right ideals of $S$ with inner morphisms. We shall term it the Schützenberger category of $S$ and denote it $\mathbb{D}(S)$ (because of its connection with Green's $\mathscr{D}$ relation). The reason for the name is that the notion of inner morphism generalizes the key notion in the definition of the Schützenberger group. Also, the relationship of the 
Schützenberger category to the Karoubi envelope can be viewed as an extension of the relationship of Schützenberger groups to maximal subgroups.

The Schützenberger category $\mathbb{D}(S)$ of a semigroup $S$ is defined as follows. The object set of $\mathbb{D}(S)$ is $S$. A morphism from $t$ to $s$ is a triple $(s, u, t)$ such that $u \in s S^{1} \cap S^{1} t$. The composition of morphisms is given by $(s, x t, t)(t, t y, r)=(s, x t y, r)$ (with $\left.x, y \in S^{1}\right)$, which the reader readily verifies is well defined. The identity at $s$ is $(s, s, s)$.

Remark 3.3. The category $\mathbb{K}(S)$ is the full subcategory of $\mathbb{D}(S)$ whose objects are the idempotents of $S$. Indeed, $(e, x f, f)(f, f y, g)=(e, x f y, g)=$ $(e, x f f y, g)$ when $e, f$ are idempotents.

Remark 3.4. The local monoids of the Schützenberger category has been studied by Diekert and his collaborators under the name local divisors and put to good effect; see for example [7-9]. The Schützenberger category is novel to this paper.

Theorem 3.5. Let $S$ be a semigroup. Then $\mathbb{D}(S)$ is equivalent to the category of principal right ideals of $S$ and inner morphisms via the functor given on objects by $s \mapsto s S^{1}$.

Proof. Let $C$ be the latter category and define a functor $F: \mathbb{D}(S) \rightarrow C$ on objects by $s \mapsto s S^{1}$ and on arrows by $(s, u t, t) \mapsto(x \mapsto u x)$ for $u \in S^{1}$. First note that this is well defined because if $u t=v t$, then $u x=v x$ for all $x \in t S^{1}$. Also it follows from Proposition 3.1 that $x \mapsto u x$ is an inner morphism. The mapping $F$ is surjective on objects and is a bijection on hom sets by Proposition 3.1. It remains to prove that $F$ preserves identities and composition. We have that $F(s, s, s)=F(s, 1 s, s)=(x \mapsto x)$ and so $F$ preserves identities. Consider composable arrows $(s, a, t),(t, b, v)$ and write $a=u t$ and $b=t y=z v$. Then $F(s, a, t) F(t, b, v)(x)=u z x$. On the other hand $(s, a, t)(t, b, v)=(s, u t y, v)=(s, u z v, v)$. But $F(s, u z v, v)(x)=u z x$ and so $F$ is a functor. This completes the proof.

Trivially, $\mathbb{D}(S)^{o p}=\mathbb{D}\left(S^{o p}\right)$, from which we obtain that the category of principal left ideals of $S$ and inner morphisms is dual to the category of principal right ideals of $S$ and inner morphisms. It thus follows from Proposition 3.1, and the fact that dualizing a category preserves automorphism groups, that the left and right Schützenberger groups of an $\mathscr{H}$-class are isomorphic.

As a consequence of Theorem 3.5 (and its proof) and Proposition 3.1, we obtain the following lemma.

Lemma 3.6. Let $s, t, u \in S$. The triple $(s, u, t)$ is an isomorphism of $\mathbb{D}(S)$ if and only if $s \mathscr{R} u \mathscr{L} t$. In particular, $s$ and $t$ are isomorphic objects of $\mathbb{D}(S)$ if and only if they are $\mathscr{D}$-equivalent elements of $S$. 
We shall use Lemma 3.6 frequently, often without explicit reference. A consequence of this lemma is that the inclusion $\mathbb{K}(S) \rightarrow \mathbb{D}(S)$ is an equivalence if and only if $S$ is regular. Another consequence of Theorem 3.5 and Proposition 3.1 is the following lemma.

Lemma 3.7. Let $s \in S$. The Schützenberger group of $s$ in $S$ is isomorphic to the automorphism group of $s$ in the category $\mathbb{D}(S)$.

We mention that the Schützenberger category has some properties in common with another category built from $S$, introduced in [13]. Despite their similarities, there are important differences between these two categories. For example, when $S$ is stable (e.g., if $S$ is finite or compact; see [18, Appendix]) all endomorphisms in the category defined in [13] are automorphisms.

\section{Lifting a functor Between Karoubi envelopes to SCHÜTZENBERGER CATEGORIES}

Given a category $C$ and a semigroup $T$, a semi-functor $F: C \rightarrow T$ is a mapping from the arrows of $C$ to $T$ satisfying $F(f g)=F(f) F(g)$ whenever $f, g$ are composable arrows. There is no requirement on what $F$ does to identities.

Let $\gamma_{D}: \mathbb{D}(T) \rightarrow T$ be the map sending a morphism $(s, u, t)$ to $u$. We do not claim that $\gamma_{D}$ is a semi-functor, however the restriction $\gamma_{K}$ of $\gamma_{D}$ to $\mathbb{K}(T)$ is a faithful semi-functor (that is, injective on hom-sets). If $F$ is a functor from a category $C$ into $\mathbb{D}(T)$, we denote by $F_{m}$ the composition $\gamma_{D} \circ F$. We also use the notation $F_{m}$ for a composition of the form $\gamma_{K} \circ F$. The subscript $m$ stands for middle, because if $\alpha$ is a morphism of $C$ then $F_{m}(\alpha)$ is the second component of the triple $F(\alpha)$.

The definition of the $\mathscr{J}$-order on a semigroup can easily be extended to categories: if $f, g$ are arrows of a category $C$, then $f \leq \mathscr{J} g$ if there are arrows $a, b$ with $f=a g b$. Similar remarks apply to the $\mathscr{L}$ - and $\mathscr{R}$-orders and the $\mathscr{D}$-relation.

Proposition 4.1. Let $F: C \rightarrow D$ be an equivalence of categories. Then $F$ reflects the $\mathscr{J}$-order, that is, $F(f) \leq \mathscr{J} F(g)$ if and only if $f \leq \mathscr{J} g$.

Proof. If $f=a g b$, then $F(f)=F(a) F(g) F(b)$. This establishes the first statement. Suppose that $G$ is a quasi-inverse of $F$ and let $\eta: 1_{C} \Rightarrow G F$ be a natural isomorphism. If $F(f) \leq \mathscr{J} F(g)$, then we can find arrows $a, b$ of $D$ with $a F(g) b=F(f)$. Thus $G(a) G F(g) G(b)=G F(f)$, that is, $G F(f) \leq \mathscr{J} G F(g)$. It thus suffices to observe that, for any arrow $x: c \rightarrow d$ of $C$, one has $x \mathscr{J} G F(x)$. But commutativity of

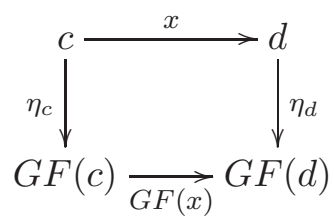


implies that $x=\eta_{d}^{-1} G F(x) \eta_{c}$ and $G F(x)=\eta_{d} x \eta_{c}^{-1}$, thereby completing the proof.

Our next proposition shows how the $\mathscr{J}$-order of $S$ is encoded in $\mathbb{D}(S)$ if $S$ is a semigroup with local units.

Proposition 4.2. Let $S$ be a semigroup with local units and $s, t \in S$. Then the following are equivalent:

(1) $s \leq \mathscr{f} t$;

(2) $(a, s, b) \leq \mathscr{g}(c, t, d)$ in $\mathbb{D}(S)$ for some $a, b, c, d \in S$;

(3) $(a, s, b) \leq \mathscr{J}(c, t, d)$ in $\mathbb{D}(S)$ for all $a, b, c, d \in S$ with as $=s=s b$ and $c t=t=t d$.

Proof. Trivially (3) implies (2) since $S$ has local units. To see that (2) implies $(1)$, let $(a, s, b)=(a, u, c)(c, t, d)(d, v, b)$. Then we can write $u=x c$ and $v=d y$ with $x, y \in S^{1}$. Then $s=x t y$ and so $s \leq \mathscr{J} t$. Suppose that (1) holds, say $s=x t y$ with $x, y \in S^{1}$. Let $a, b, c, d$ be as in (3). Then $(a, a x c, c)(c, t, d)(d, d y b, b)=(a, a x t y b, b)$. But $a x t y b=a s b=s$, so $(3)$ holds.

Definition 4.3. Let $S$ and $T$ be semigroups with local units. We say that a functor $F: \mathbb{D}(S) \rightarrow \mathbb{D}(T)$ is good if

(1) $F$ restricts to an equivalence $\mathbb{K}(S) \rightarrow \mathbb{K}(T)$;

(2) for all $s \in S$, there exist idempotents $e_{s}, f_{s}$ with $e_{s} s f_{s}=s$ and

$$
\begin{aligned}
& F\left(e_{s}, s, s\right)=\left(F\left(e_{s}\right), F(s), F(s)\right), \\
& F\left(s, s, f_{s}\right)=\left(F(s), F(s), F\left(f_{s}\right)\right) .
\end{aligned}
$$

Let us say that a functor $F: \mathbb{D}(S) \rightarrow \mathbb{D}(T)$ reflects regularity if $F(s)$ is regular if and only if $s$ is regular for $s \in S$. We say that it reflects the $\mathscr{J}$-order on objects if $s \leq \mathscr{J} s^{\prime}$ if and only only if $F(s) \leq \mathscr{J} F\left(s^{\prime}\right)$ for all $s, s^{\prime} \in S$.

Lemma 4.4. Let $S$ and $T$ be semigroups with local units and let $F: \mathbb{D}(S) \rightarrow$ $\mathbb{D}(T)$ be a good functor. Then $F$ is an equivalence reflecting regularity and the $\mathscr{J}$-order on objects.

Proof. Fix, for each $s \in S$, idempotents $e_{s}, f_{s}$ as in the definition of a good functor. First we show that $F$ is an equivalence. To prove that $F$ is faithful suppose that $F(a, s, b)=F\left(a, s^{\prime}, b\right)$. Choose idempotents $e, f \in E(S)$ with $e a=a$ and $b f=b$. Then since $(e, s, f)=(e, a, a)(a, s, b)(b, b, f)$ and $\left(e, s^{\prime}, f\right)=(e, a, a)\left(a, s^{\prime}, b\right)(b, b, f)$ it follows that $F(e, s, f)=F\left(e, s^{\prime}, f\right)$ and so $s=s^{\prime}$ since $\left.F\right|_{\mathbb{K}(S)}$ is faithful. Thus $F$ is faithful.

To see that $F$ is full, let $(F(a), t, F(b))$ be a morphism of $\mathbb{D}(T)$. From $\left(e_{s}, s, f_{s}\right)=\left(e_{s}, s, s\right)\left(s, s, f_{s}\right)$, we obtain that

$$
\begin{aligned}
F\left(e_{s}, s, f_{s}\right) & =\left(F\left(e_{s}\right), F(s), F(s)\right)\left(F(s), F(s), F\left(f_{s}\right)\right) \\
& =\left(F\left(e_{s}\right), F(s), F\left(f_{s}\right)\right)
\end{aligned}
$$


for all $s \in S$. By definition of $\mathbb{D}(T)$, we have $t=F(a) x=y F(b)$ for some $x, y \in T^{1}$. Because $F\left(e_{c}, c, f_{c}\right)$ is a morphism of $\mathbb{K}(T)$ for $c=a, b$, we have $t=F(a) F\left(f_{a}\right) x F\left(f_{b}\right)$, and so without loss of generality we may assume that $x=F\left(f_{a}\right) x F\left(f_{b}\right)$. Since $\left.F\right|_{\mathbb{K}(S)}$ is full, there is $x^{\prime}$ such that $F\left(f_{a}, x^{\prime}, f_{b}\right)=\left(F\left(f_{a}\right), x, F\left(f_{b}\right)\right)$. Similarly, we can assume $y=F\left(e_{a}\right) y F\left(e_{b}\right)$ and find $y^{\prime}$ such that $F\left(e_{a}, y^{\prime}, e_{b}\right)=\left(F\left(e_{a}\right), y, F\left(e_{b}\right)\right)$. Now

$$
\begin{aligned}
F\left(e_{a}, a x^{\prime}, f_{b}\right) & =F\left(e_{a}, a, f_{a}\right) F\left(f_{a}, x^{\prime}, f_{b}\right) \\
& =\left(F\left(e_{a}\right), F(a), F\left(f_{a}\right)\right)\left(F\left(f_{a}\right), x, F\left(f_{b}\right)\right)=\left(F\left(e_{a}\right), t, F\left(f_{b}\right)\right),
\end{aligned}
$$

and similarly $F\left(e_{a}, y^{\prime} b, f_{b}\right)=\left(F\left(e_{a}\right), t, F\left(f_{b}\right)\right)$. Thus, since $\left.F\right|_{\mathbb{K}(S)}$ is faithful, we conclude $a x^{\prime}=y^{\prime} b$. Therefore, $\left(a, a x^{\prime}, b\right)$ is a morphism of $\mathbb{D}(S)$. Let $F\left(a, a x^{\prime}, b\right)=(F(a), v, F(b))$. Now $\left(e_{a}, a, a\right)\left(a, a x^{\prime}, b\right)\left(b, b, f_{b}\right)=\left(e_{a}, a x^{\prime}, f_{b}\right)$ and the definition of a good functor imply that

$$
\begin{aligned}
\left(F\left(e_{a}\right), t, F\left(f_{b}\right)\right) & =F\left(e_{a}, a x^{\prime}, f_{b}\right) \\
& =\left(F\left(e_{a}\right), F(a), F(a)\right) F\left(a, a x^{\prime}, b\right)\left(F(b), F(b), F\left(e_{b}\right)\right) \\
& =\left(F\left(e_{a}\right), v, F\left(f_{b}\right)\right) .
\end{aligned}
$$

Thus $v=t$ and $(F(a), t, F(b))=F\left(a, a x^{\prime}, b\right)$, and so we conclude that $F$ is full.

Finally, we prove that $F$ is essentially surjective. Let $t$ be an element of $T$. Then $t=e_{0} t f_{0}$ for some idempotents $e_{0}, f_{0} \in E(T)$. Since $\left.F\right|_{\mathbb{K}(S)}$ is essentially surjective, there are idempotents $e$ and $f$ of $S$ such that $e_{0} \mathscr{D} F(e)$ and $f_{0} \mathscr{D} F(f)$. Therefore, there are $x$ and $y$ in the $\mathscr{D}$-class of $e_{0}$ such that $e_{0}=x y$ and $F(e)=y x$, and there are $x^{\prime}$ and $y^{\prime}$ in the $\mathscr{D}$-class of $f_{0}$ such that $f_{0}=x^{\prime} y^{\prime}$ and $F(f)=y^{\prime} x^{\prime}$. Let $t^{\prime}=y t x^{\prime}$. Then $t^{\prime}=y e_{0} t f_{0} x^{\prime}=y x y t x^{\prime} y^{\prime} x^{\prime}=$ $F(e) t^{\prime} F(f)$, thus $\left(F(e), t^{\prime}, F(f)\right)$ is a morphism of $\mathbb{K}(T)$. Since $F$ is full, there is $s \in S$ such that $\left(F(e), t^{\prime}, F(f)\right)=F(e, s, f)$.

We have $(e, s, f)=\left(e, e e_{s}, e_{s}\right)\left(e_{s}, s, f\right)$ and $\left(e_{s}, s, f\right)=\left(e_{s}, e_{s} e, e\right)(e, s, f)$ and so $(e, s, f) \mathscr{L}\left(e_{s}, s, f\right)$ in $\mathbb{K}(S)$. Also, $\left(e_{s}, s, f\right)=\left(e_{s}, s, f_{s}\right)\left(f_{s}, f_{s} f, f\right)$ and $\left(e_{s}, s, f_{s}\right)=\left(e_{s}, s, f\right)\left(f, f f_{s}, f_{s}\right)$, thus $\left(e_{s}, s, f\right) \mathscr{R}\left(e_{s}, s, f_{s}\right)$ in $\mathbb{K}(S)$. It follows that $(e, s, f) \mathscr{D}\left(e_{s}, s, f_{s}\right)$ in $\mathbb{K}(S)$. Hence, using (4.1), we have

$$
\left(F\left(e_{s}\right), F(s), F\left(f_{s}\right)\right)=F\left(e_{s}, s, f_{s}\right) \mathscr{D} F(e, s, f)=\left(F(e), t^{\prime}, F(f)\right)
$$

(in $\mathbb{K}(T)$ ) and so $F(s) \mathscr{D} t^{\prime}$ by applying the semi-functor $\gamma_{K}: \mathbb{K}(T) \rightarrow T$ that projects to the middle coordinate. Since $x y t=t$ and $y t=t^{\prime} y^{\prime}$, we have $t \mathscr{L}$ yt $\mathscr{R} t^{\prime}$. Therefore, $t$ and $F(s)$ are $\mathscr{D}$-equivalent and hence isomorphic in $\mathbb{D}(T)$. This proves that $F$ is essentially surjective.

To prove that $F$ reflects regularity, we shall use freely that Lemma 3.6 implies that an element of a semigroup $X$ is regular if and only if it is isomorphic in $\mathbb{D}(X)$ to an element of $\mathbb{K}(X)$. Suppose that $s \in S$ is regular. Then $s$ is isomorphic to some object $e$ of $\mathbb{K}(S)$ and hence $F(s)$ is isomorphic to $F(e) \in \mathbb{K}(T)$. Thus $F(s)$ is regular. Conversely, if $F(s)$ is regular, then $F(s)$ is isomorphic to some object of $\mathbb{K}(T)$ and hence, since the restriction $F: \mathbb{K}(S) \rightarrow \mathbb{K}(T)$ is an equivalence, to some object of $F(\mathbb{K}(S))$. Since 
equivalences reflect isomorphisms, it follows that $s$ is isomorphic to some object of $\mathbb{K}(S)$ and so $s$ is regular.

To prove $F$ reflects the $\mathscr{J}$-order on $S$, let $s, s^{\prime} \in S$. By (4.1) we have

$$
\begin{aligned}
F\left(e_{s}, s, f_{s}\right) & =\left(F\left(e_{s}\right), F(s), F\left(f_{s}\right)\right) \\
F\left(e_{s^{\prime}}, s^{\prime}, f_{s^{\prime}}\right) & =\left(F\left(e_{s^{\prime}}\right), F\left(s^{\prime}\right), F\left(f_{s^{\prime}}\right)\right) .
\end{aligned}
$$

Note that $F\left(e_{s}, s, f_{s}\right), F\left(e_{s^{\prime}}, s^{\prime}, f_{s^{\prime}}\right) \in \mathbb{K}(T)$. By Propositions 4.1 and 4.2 we deduce

$$
\begin{aligned}
s \leq \mathscr{J} s^{\prime} & \Longleftrightarrow\left(e_{s}, s, f_{s}\right) \leq \mathscr{J}\left(e_{s^{\prime}}, s^{\prime}, f_{s^{\prime}}\right) \\
& \Longleftrightarrow F\left(e_{s}, s, f_{s}\right) \leq \mathscr{J} F\left(e_{s^{\prime}}, s^{\prime}, f_{s^{\prime}}\right) \Longleftrightarrow F(s) \leq_{\mathscr{J}} F\left(s^{\prime}\right) .
\end{aligned}
$$

This completes the proof.

Consider a functor $F: \mathbb{K}(S) \rightarrow \mathbb{K}(T)$, for a pair of semigroups $S$ and $T$ with local units. Our goal is to lift $F$ to a functor $\mathbb{D}(S) \rightarrow \mathbb{D}(T)$. Moreover, if $F$ is an equivalence, the lifting will be a good functor.

Let $\left(e_{s}\right)_{s \in S}$ and $\left(f_{s}\right)_{s \in S}$ be families of idempotents of $S$ such that $s=$ $e_{s} s f_{s}$, for each $s \in S$, and such that if $\varepsilon$ is an idempotent of $S$ then $e_{\varepsilon}=f_{\varepsilon}=$ $\varepsilon$. We will use without comment that $s S^{1} \cap S^{1} t \subseteq e_{s} S f_{t}$ for all $s, t \in S$. We define a functor $\widehat{F}: \mathbb{D}(S) \rightarrow \mathbb{D}(T)$ sending an object $s$ of $\mathbb{D}(S)$ to $F_{m}\left(e_{s}, s, f_{s}\right)$ and a morphism $(s, u, t)$ to $\left(\widehat{F}(s), F_{m}\left(e_{s}, u, f_{t}\right), \widehat{F}(t)\right)$. One must show that the latter triple is indeed an element of $\mathbb{D}(T)$, a task which we include in the proof of Theorem 4.5 .

Theorem 4.5. Suppose that $S$ and $T$ are semigroups with local units and let $F: \mathbb{K}(S) \rightarrow \mathbb{K}(T)$ be a functor. Then $\widehat{F}: \mathbb{D}(S) \rightarrow \mathbb{D}(T)$, defined above, is a functor whose restriction to $\mathbb{K}(S)$ coincides with $F$. Moreover, if $F$ is an equivalence, then $\widehat{F}$ is a good functor, and hence is an equivalence reflecting regularity and the $\mathscr{J}$-order on objects.

Proof. If $\varepsilon$ and $\phi$ are idempotents of $S$ then

$$
\widehat{F}(\varepsilon, u, \phi)=\left(F_{m}\left(e_{\varepsilon}, \varepsilon, f_{\varepsilon}\right), F_{m}\left(e_{\varepsilon}, u, f_{\phi}\right), F_{m}\left(e_{\phi}, \phi, f_{\phi}\right)\right)=F(\varepsilon, u, \phi),
$$

because the restrictions of the maps $s \mapsto e_{s}$ and $s \mapsto f_{s}$ to $E(S)$ are the identities. Therefore, the restriction of $\widehat{F}$ to $\mathbb{K}(S)$ coincides with $F$.

We now show that $\widehat{F}(s, u, t)$ is indeed a morphism of $\mathbb{D}(S)$. Let $x \in$ $S^{1}$ be such that $u=s x$. Since $s=s f_{s}$ and $u=u f_{t}$, we may as well suppose that $x=f_{s} x f_{t}$. Then $\left(e_{s}, u, f_{t}\right)=\left(e_{s}, s, f_{s}\right)\left(f_{s}, x, f_{t}\right)$ in $\mathbb{K}(S)$. Since the restriction of $F_{m}$ to $\mathbb{K}(S)$ is a semi-functor, we conclude that $\widehat{F}(s)=F_{m}\left(e_{s}, s, f_{s}\right) \geq \mathscr{R} F_{m}\left(e_{s}, u, f_{t}\right)$. Similarly, $\widehat{F}(t) \geq \mathscr{L} F_{m}\left(e_{s}, u, f_{t}\right)$. It follows that $\left(\widehat{F}(s), F_{m}\left(e_{s}, u, f_{t}\right), \widehat{F}(t)\right)$ is a morphism of $\mathbb{D}(T)$.

Next, we prove that $\widehat{F}$ is a functor. Clearly, $\widehat{F}\left(1_{s}\right)=(\widehat{F}(s), \widehat{F}(s), \widehat{F}(s))=$ $1_{\widehat{F}(s)}$ by construction. Let $(s, u, t)$ and $(t, v, r)$ be two composable arrows of $\mathbb{D}(S)$. Let $x$ be such that $u=x t$. We may suppose that $x=e_{s} x e_{t}$. Then

$$
\widehat{F}((s, u, t)(t, v, r))=\widehat{F}(s, x v, r)=\left(\widehat{F}(s), F_{m}\left(e_{s}, x v, f_{r}\right), \widehat{F}(r)\right) .
$$


On the other hand, from $\left(e_{s}, u, f_{t}\right)=\left(e_{s}, x, e_{t}\right)\left(e_{t}, t, f_{t}\right)$, we have $F_{m}\left(e_{s}, u, f_{t}\right)=$ $F_{m}\left(e_{s}, x, e_{t}\right) \cdot \widehat{F}(t)$ and so

$$
\begin{aligned}
\widehat{F}(s, u, t) \cdot \widehat{F}(t, v, r) & =\left(\widehat{F}(s), F_{m}\left(e_{s}, u, f_{t}\right), \widehat{F}(t)\right) \cdot\left(\widehat{F}(t), F_{m}\left(e_{t}, v, f_{r}\right), \widehat{F}(r)\right) . \\
& =\left(\widehat{F}(s), F_{m}\left(e_{s}, x, e_{t}\right) F_{m}\left(e_{t}, v, f_{r}\right), \widehat{F}(r)\right) .
\end{aligned}
$$

Comparing (4.2) and (4.3), we obtain $\widehat{F}((s, u, t)(t, v, r))=\widehat{F}(s, u, t) \cdot \widehat{F}(t, v, r)$. Thus $\widehat{F}$ is a functor.

It remains to prove that if $F$ is an equivalence, then $\widehat{F}$ is a good functor. But this is immediate from the definition since

$$
\widehat{F}\left(e_{s}, s, s\right)=\left(\widehat{F}\left(e_{s}\right), F_{m}\left(e_{s}, s, f_{s}\right), \widehat{F}(s)\right)=\left(\widehat{F}\left(e_{s}\right), \widehat{F}(s), \widehat{F}(s)\right)
$$

using that $e_{e_{s}}=e_{s}$. Similarly $\widehat{F}\left(s, s, f_{s}\right)=\left(\widehat{F}(s), \widehat{F}(s), \widehat{F}\left(f_{s}\right)\right)$. Thus $F$ is good, completing the proof in light of Lemma 4.4 .

Corollary 4.6. Let $S$ and $T$ be semigroups with local units. If $\mathbb{K}(S)$ and $\mathbb{K}(T)$ are equivalent, then so are $\mathbb{D}(S)$ and $\mathbb{D}(T)$.

We do not know if the converse of Corollary 4.6 holds.

\section{ACtions of $\mathbb{K}(S)$ AND $\mathbb{D}(S)$}

From here on out, we mention only right actions, and so action will be synonymous of right action until the end of this paper.

An action of a small category $C$ on a set $Q$ is a contravariant functor $\mathbb{A}: C \rightarrow$ Set such that $\mathbb{A}(c)$ is a subset of $Q$ for every object $c$ of $C$, i.e., a presheaf on $C$ taking values in subsets of $Q$ [17]. If $s: c \rightarrow d$ is a morphism of $C$, we may use the notation $q \cdot s$ for $\mathbb{A}(s)(q)$, where $q \in \mathbb{A}(d)$, and the notation $\mathbb{A}(d) \cdot s$ for the image of the function $\mathbb{A}(s): \mathbb{A}(d) \rightarrow \mathbb{A}(c)$. The notation $q \cdot s$ and $\mathbb{A}(d) \cdot s$ may be simplified to $q s$ and $\mathbb{A}(d) s$.

Definition 5.1 (Equivalent actions). Consider two actions $\mathbb{A}: C \rightarrow$ Set and $\mathbb{A}^{\prime}: D \rightarrow$ Set. We say that $\mathbb{A}$ and $\mathbb{A}^{\prime}$ are equivalent, and write $\mathbb{A} \sim \mathbb{A}^{\prime}$, if there is an equivalence $F: C \rightarrow D$ such that $\mathbb{A}$ and $\mathbb{A}^{\prime} \circ F$ are isomorphic functors.

Remark 5.2. Note that the binary relation $\sim$ is an equivalence relation on the class of actions. It is clearly reflexive; transitivity and symmetry follow straightforwardly from the fact that, for small categories $C$ and $D$, if $F: C \rightarrow$ $D$ and $G: C \rightarrow D$ are isomorphic functors, then $F H$ and $G H$ are also isomorphic for every functor $H$ from a category into $C$.

Definition 5.3. Consider an action of a semigroup $S$ on a set $Q$. Let $\mathbb{A}_{Q}$ be the action of $\mathbb{K}(S)$ on $Q$ such that $\mathbb{A}_{Q}(e)=Q e$ for every object $e$ of $\mathbb{K}(S)$, and such that $\mathbb{A}_{Q}((e, s, f))$ is the function $Q e \rightarrow Q f$ mapping $q$ to $q s$, for every $q \in Q e$; that is, $q \cdot(e, s, f)=q \cdot s$.

Similarly, let $\mathbb{B}_{Q}$ be the action of $\mathbb{D}(S)$ on $Q$ such that $\mathbb{B}_{Q}(s)=Q s$ for each object $s$ of $\mathbb{D}(S)$, and where $\mathbb{B}_{Q}((s, u, t))$ is the function $Q s \rightarrow Q t$ mapping $q s$ to $q u$, when $q \in Q$. That is, $q s \cdot(s, u, t)=q u$. Note that $\mathbb{B}_{Q}((s, u, t))$ 
is well defined: indeed, for all $q_{1}, q_{2} \in Q$, if $q_{1} s=q_{2} s$, writing $u=s x$ with $x \in S^{1}$, we have $q_{1} u=q_{1} s x=q_{2} s x=q_{2} u$. Note also that the restriction of $\mathbb{B}_{Q}$ to $\mathbb{K}(S)$ is $\mathbb{A}_{Q}$.

Lemma 5.4. If the action of the semigroup $S$ on $Q$ is faithful, then the actions $\mathbb{A}_{Q}$ and $\mathbb{B}_{Q}$ are faithful.

Proof. Let $(s, u, t),(s, v, t)$ be distinct coterminal morphisms of $\mathbb{D}(S)$. By faithfulness for $S$ we can find $q \in Q$ with $q u \neq q v$. Then, by the definition of $\mathbb{B}_{Q}$, we have $q s(s, u, t)=q u$ and $q s(s, v, t)=q v$. We conclude $\mathbb{B}_{Q}$ is faithful. As $\mathbb{A}_{Q}$ is the restriction of $\mathbb{B}_{Q}$ to $\mathbb{K}(S)$, the action $\mathbb{A}_{Q}$ is also faithful.

The remaining part of this section concerns the lifting of an equivalence $\mathbb{A}_{Q} \sim \mathbb{A}_{R}$ to an equivalence $\mathbb{B}_{Q} \sim \mathbb{B}_{R}$. Theorem 4.5 will be crucial. The following proposition deals with other technicalities that need to be addressed.

Proposition 5.5. Let $S$ and $T$ be semigroups with local units. Suppose that $S$ acts on the set $Q$ and $T$ acts on the set $R$. Let $F: \mathbb{K}(S) \rightarrow \mathbb{K}(T)$ be a functor and $\eta: \mathbb{A}_{Q} \Rightarrow \mathbb{A}_{R} \circ F$ a natural transformation. Retaining the notation of Theorem 4.5, if $s \in S$ and $q \in Q$, then $\eta_{f_{s}}(q s)=\eta_{e_{s}}\left(q e_{s}\right)$. $\widehat{F}(s)$. Therefore, the map $\eta_{f_{s}}: Q \cdot f_{s} \rightarrow R \cdot F\left(f_{s}\right)$ restricts to a mapping $\lambda_{s}: Q \cdot s \rightarrow R \cdot \widehat{F}(s)$. Moreover, the family $\lambda=\left(\lambda_{s}\right)_{s \in S}$ constitutes a natural transformation $\lambda: \mathbb{B}_{Q} \Rightarrow \mathbb{B}_{R} \circ \widehat{F}$. Furthermore, if $\eta$ is a natural isomorphism, then so is $\lambda$.

Proof. Note that, for all $s \in S$, we have $Q \cdot s \subseteq Q \cdot f_{s}$. Moreover, since $\eta$ is a natural transformation, the following chain of equalities holds for each $q \in Q$ :

$$
\eta_{f_{s}}(q s)=\eta_{f_{s}}\left(q e_{s} \cdot\left(e_{s}, s, f_{s}\right)\right)=\eta_{e_{s}}\left(q e_{s}\right) \cdot F\left(e_{s}, s, f_{s}\right)=\eta_{e_{s}}\left(q e_{s}\right) \cdot \widehat{F}(s) .
$$

Hence, we may restrict the map $\eta_{f_{s}}$ to a map $\lambda_{s}: Q \cdot s \rightarrow R \cdot \widehat{F}(s)$. We wish to show that the family $\lambda=\left(\lambda_{s}\right)_{s \in S}$ yields a natural transformation $\lambda: \mathbb{B}_{Q} \Rightarrow \mathbb{B}_{R} \circ \widehat{F}$. That is, we want to prove that the following diagram is commutative

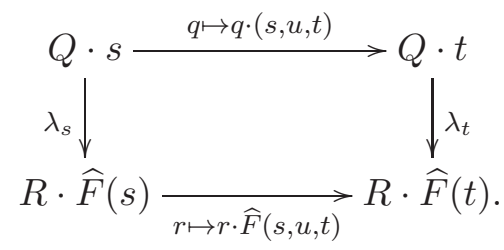

for each arrow $(s, u, t)$ of $\mathbb{D}(S)$. Let $q$ be an element of $Q$. Then $\lambda_{s}(q s)=$ $\eta_{e_{s}}\left(q e_{s}\right) \widehat{F}(s)$ by (5.1). We then have the following chain of equalities, equality (5.3) holding by the definition of $\widehat{F}$ and equality (5.4) because $\eta$ is a 
natural transformation:

$$
\begin{aligned}
\lambda_{s}(q s) \cdot \widehat{F}(s, u, t) & =\eta_{e_{s}}\left(q e_{s}\right) \widehat{F}(s) \cdot \widehat{F}(s, u, t) \\
& =\eta_{e_{s}}\left(q e_{s}\right) \widehat{F}(s) \cdot\left(\widehat{F}(s), F_{m}\left(e_{s}, u, e_{t}\right), \widehat{F}(t)\right) \\
& =\eta_{e_{s}}\left(q e_{s}\right) \cdot F_{m}\left(e_{s}, u, f_{t}\right) \\
& =\eta_{e_{s}}\left(q e_{s}\right) \cdot F\left(e_{s}, u, f_{t}\right) \\
& =\eta_{f_{t}}\left(q e_{s} \cdot\left(e_{s}, u, f_{t}\right)\right) \\
& =\eta_{f_{t}}(q u) .
\end{aligned}
$$

Since $q u \in Q t$, we have $\eta_{f_{t}}(q u)=\lambda_{t}(q u)$, thus we have shown

$$
\lambda_{s}(q s) \cdot \widehat{F}(s, u, t)=\lambda_{t}(q u) .
$$

By the definition of the action of $\mathbb{D}(S)$ on $Q$, we also have $q s \cdot(s, u, t)=$ $q u$. Therefore $\lambda_{s}(q s) \cdot \widehat{F}(s, u, t)=\lambda_{t}(q s \cdot(s, u, t))$, which establishes the commutativity of (5.2).

Finally, suppose that $\eta$ is a natural isomorphism. For every object $s$ of $\mathbb{D}(S)$, the function $\lambda_{s}$ is obviously injective, since it is a restriction of the injective function $\eta_{e_{s}}$. It remains to prove that $\lambda_{s}$ is onto. Let $r \in R$. Then we have

$$
r \widehat{F}(s)=r F_{m}\left(e_{s}, s, f_{s}\right)=r F\left(e_{s}\right) \cdot F\left(e_{s}, s, f_{s}\right) .
$$

Since $\eta_{e_{s}}$ is onto, there is $q \in Q e_{s}$ such that $r F\left(e_{s}\right)=\eta_{e_{s}}(q)$. As $\eta$ is a natural transformation, it then follows from (5.5) that $r \widehat{F}(s)=\eta_{f_{s}}\left(q \cdot\left(e_{s}, s, f_{s}\right)\right)=$ $\lambda_{s}(q s)$. Therefore $\lambda_{s}$ is onto.

Corollary 5.6. Let $S$ and $T$ be semigroups with local units with actions on $Q$ and $R$, respectively. If $\mathbb{A}_{Q}$ and $\mathbb{A}_{R}$ are equivalent actions, then there is an equivalence $G: \mathbb{D}(S) \rightarrow \mathbb{D}(T)$ and a natural isomorphism $\lambda: \mathbb{B}_{Q} \Rightarrow \mathbb{B}_{R} \circ G$ such that

(1) $G$ restricts to an equivalence $\mathbb{K}(S) \rightarrow \mathbb{K}(T)$;

(2) $G$ reflects regularity and the $\mathscr{J}$-order on objects;

(3) $\lambda$ restricts to a natural isomorphism $\left.\mathbb{A}_{Q} \Rightarrow \mathbb{A}_{R} \circ G\right|_{\mathbb{K}(S)}$.

Proof. This is an immediate consequence of Theorem4.5 and Proposition 5.5: just take $G=\widehat{F}$, where $F$ is an equivalence $\mathbb{K}(S) \rightarrow \mathbb{K}(T)$ such that $\mathbb{A}_{Q}$ and $\mathbb{A}_{R} \circ F$ are isomorphic.

In particular, we have the following.

Corollary 5.7. Let $S$ and $T$ be semigroups with local units with actions on $Q$ and $R$, respectively. If $\mathbb{A}_{Q} \sim \mathbb{A}_{R}$ then $\mathbb{B}_{Q} \sim \mathbb{B}_{R}$.

\section{KAROUBI INVARIANTS OF AN ACTION}

Let $C$ be a category. An assignment of an object $F(Q, S)$ of $C$ to each action of a semigroup $S$ on a set $Q$ is said to be a Karoubi invariant of the action if $F(Q, S)$ is isomorphic to $F\left(Q^{\prime}, T\right)$ whenever the actions $\mathbb{A}_{Q}$ and 
$\mathbb{A}_{Q^{\prime}}$ are equivalent. This section includes several Karoubi invariants used in [6] to obtain flow equivalence invariants for symbolic dynamical systems.

6.1. The poset of an action. Suppose that a semigroup $S$ acts on a set $Q$. Let $I=Q \cdot E(S)$ and define a preorder $\preceq$ on $I$ by $q \preceq q^{\prime}$ if $q^{\prime} L U(S) \subseteq q L U(S)$, that is, $q^{\prime}=q s$ for some $s \in L U(S)$. As usual, define $q \sim q^{\prime}$ if $q \preceq q^{\prime}$ and $q^{\prime} \preceq q$. Then $P(Q)=I / \sim$ is a poset, which can be identified with the poset of cyclic $L U(S)$-subsets $\{q \cdot L U(S) \mid q \in I\}$ ) ordered by reverse inclusion. We show that $P(Q)$ is a Karoubi invariant of the action of $S$ on $Q$. More precisely, we have the following result.

Theorem 6.1. Suppose $S, T$ are semigroups acting on sets $Q, Q^{\prime}$, respectively. Suppose that $F: \mathbb{K}(S) \rightarrow \mathbb{K}(T)$ is an equivalence of categories and that $\eta: \mathbb{A}_{Q} \Rightarrow \mathbb{A}_{Q^{\prime}} \circ F$ is an isomorphism. Then there is a well-defined isomorphism $f: P(Q) \rightarrow P\left(Q^{\prime}\right)$ of posets given by $f(q L U(S))=\eta_{e}(q) L U(T)$ for $q \in Q e$. In particular, $P(Q)$ is a Karoubi invariant.

Proof. We claim that if $q \in Q e$ and $q^{\prime} \in Q e^{\prime}$ with $e, e^{\prime} \in E(S)$, then $q^{\prime} \in$ $q L U(S)$ if and only if there exists an arrow $\left(e, s, e^{\prime}\right)$ of $\mathbb{K}(S)$ with $q^{\prime}=$ $q\left(e, s, e^{\prime}\right)$. Indeed, if $q^{\prime}=q s$ with $s \in L U(S)$, then $q^{\prime}=q^{\prime} e^{\prime}=q s e^{\prime}=q e s e^{\prime}=$ $q\left(e, e s e^{\prime}, e^{\prime}\right)$. Conversely, if $q^{\prime}=q\left(e, s, e^{\prime}\right)$, then $q^{\prime}=q s$ and $s=e s e^{\prime} \in$ $L U(S)$ and so $q^{\prime} \in q L U(s)$. A similar claim, of course, holds for $Q^{\prime}$ and $T$.

To see that $f$ is a well-defined order preserving map, let $q, q^{\prime} \in Q E(S)$ and suppose that $q L U(S) \supseteq q^{\prime} L U(S)$. Choose $e, e^{\prime} \in E(S)$ with $q e=q$ and $q^{\prime} e^{\prime}=q^{\prime}$. Then by the claim, there is an arrow $\left(e, s, e^{\prime}\right)$ with $q\left(e, s, e^{\prime}\right)=$ $q^{\prime}$. Therefore, we have $\eta_{e^{\prime}}\left(q^{\prime}\right)=\eta_{e^{\prime}}\left(q\left(e, s, e^{\prime}\right)\right)=\eta_{e}(q) F\left(e, s, e^{\prime}\right)$ and so $\eta_{e}(q) L U(T) \supseteq \eta_{e^{\prime}}\left(q^{\prime}\right) L U(T)$ by the claim. Thus $f$ is well defined and order preserving.

To show that $f$ is an order embedding, suppose that $q e=q, q^{\prime} e^{\prime}=q^{\prime}$ (with $\left.e, e^{\prime} \in E(S)\right)$ and $\eta_{e}(q) L U(T) \supseteq \eta_{e^{\prime}}\left(q^{\prime}\right) L U(T)$. Then by the claim there is an arrow $\left(F(e), t, F\left(e^{\prime}\right)\right)$ of $\mathbb{K}(T)$ with $\eta_{e}(q)\left(F(e), t, F\left(e^{\prime}\right)\right)=\eta_{e^{\prime}}\left(q^{\prime}\right)$. Since $F$ is full, there is an arrow $\left(e, s, e^{\prime}\right)$ of $\mathbb{K}(S)$ with $F\left(e, s, e^{\prime}\right)=\left(F(e), t, F\left(e^{\prime}\right)\right)$. Then

$$
\eta_{e^{\prime}}\left(q\left(e, s, e^{\prime}\right)\right)=\eta_{e}(q)\left(F(e), t, F\left(e^{\prime}\right)\right)=\eta_{e^{\prime}}\left(q^{\prime}\right)
$$

and hence $q^{\prime}=q\left(e, s, e^{\prime}\right)$ because $\eta_{e^{\prime}}: Q e^{\prime} \rightarrow Q^{\prime} F\left(e^{\prime}\right)$ is a bijection. Thus $q L U(S) \supseteq q^{\prime} L U(S)$ by the claim. We conclude that $f$ is an order embedding.

It remains to show that $f$ is surjective. Let $q^{\prime} \in Q^{\prime} E(T)$. Choose $e^{\prime} \in E(T)$ with $q^{\prime} e^{\prime}=q^{\prime}$. Since $F$ is essentially surjective, there is an isomorphism $\left(e^{\prime}, t, F(e)\right)$ with $e \in E(S)$; say its inverse is $\left(F(e), t^{\prime}, e^{\prime}\right)$. Let $q=\eta_{e}^{-1}\left(q^{\prime}\left(e^{\prime}, t, F(e)\right)\right)$. Then $\eta_{e}(q)=q^{\prime}\left(e^{\prime}, t, F(e)\right)$ implies that $q^{\prime} L U(T) \supseteq$ $\eta_{e}(q) L U(T)$ by the claim. Conversely, we compute $\eta_{e}(q)\left(F(e), t^{\prime}, e^{\prime}\right)=$ $q^{\prime}\left(e^{\prime}, t, F(e)\right)\left(F(e), t^{\prime}, e^{\prime}\right)=q^{\prime}$ and so $\eta_{e}(q) L U(T) \supseteq q^{\prime} L U(T)$ by the claim. Thus $f(q L U(S))=\eta_{e}(q) L U(T)=q^{\prime} L U(T)$ and hence $f$ is surjective. This proves the theorem.

\subsection{Labeled preordered sets of the $\mathscr{D}$-classes.}


6.2.1. Regularity and the Schützenberger group of a $\mathscr{D}$-class are preserved by equivalences. Given a semigroup $S$, let $\mathfrak{D}(S)$ be the set of $\mathscr{D}$-classes of $S$. Endow $\mathfrak{D}(S)$ with the preorder $\preceq$ such that, if $D_{1}$ and $D_{2}$ belong to $\mathfrak{D}(S)$, then $D_{1} \preceq D_{2}$ if and only if there are $d_{1} \in D_{1}$ and $d_{2} \in D_{2}$ such that $d_{1} \leq \mathscr{J} d_{2}$. Note that if $\mathcal{D}=\mathcal{J}$ (for example, if $S$ is finite), then $\preceq$ is a partial order.

If we assign to each element $x$ of a preordered set $P$ a label $\lambda_{P}(x)$ from some set, we obtain a new structure, called labeled preordered set. A morphism in the category of labeled preordered sets is a morphism $\varphi: P \rightarrow Q$ of preordered sets such $\lambda_{Q} \circ \varphi=\lambda_{P}$.

For a semigroup $S$, assign to each element $D$ of $\mathfrak{D}(S)$ the label $\lambda(D)=$ $(\varepsilon, H)$ where $\varepsilon \in\{0,1\}$, with $\varepsilon=1$ if and only if $D$ is regular, and $H$ is the Schützenberger group of $D$. We denote the labeled preordered set thus obtained by $\mathfrak{D}_{\ell}(S)$.

Remark 6.2. Let $S$ be a semigroup, not necessarily with local units. If $\mathscr{K}$ is one of Green's relations $\mathscr{J}, \mathscr{R}$ or $\mathscr{L}$, then $s \leq \mathscr{K} t$ in $L U(S)$ if and only if $s \leq \mathscr{K} t$ in $S$, for all $s, t \in L U(S)$, and the Schützenberger group of $s \in L U(S)$ is the same in $S$ as in $L U(S)$ (cf. proof of [5. Proposition 5.2]). Therefore, $\mathfrak{D}_{\ell}(L U(S))$ is obtained from $\mathfrak{D}_{\ell}(S)$ by removing the $\mathscr{D}$-classes of $S$ not contained in $L U(S)$.

Theorem 6.3. Let $S$ and $T$ be semigroups. If $\mathbb{K}(S)$ and $\mathbb{K}(T)$ are equivalent categories, then $\mathfrak{D}_{\ell}(L U(S))$ and $\mathfrak{D}_{\ell}(L U(T))$ are isomorphic labeled preordered sets.

Before proving Theorem 6.3, we mention that, with a different language, its content is almost entirely proved in [12, Proposition 5.1], the exception being the part concerning the labeling by Schützenberger groups. Our proof fills this gap easily because of the use of the Schützenberger category.

Let $S$ and $T$ be semigroups with local units. Let $G: \mathbb{D}(S) \rightarrow \mathbb{D}(T)$ be an equivalence. Denote by $\bar{G}: \mathfrak{D}(S) \rightarrow \mathfrak{D}(T)$ the function such that $\bar{G}\left(D_{s}\right)=$ $D_{G(s)}$, where $D_{t}$ denotes the $\mathscr{D}$-class of $t$. Note that $\bar{G}$ is well defined, because $G$ preserves isomorphisms.

Lemma 6.4. Suppose that the equivalence $G: \mathbb{D}(S) \rightarrow \mathbb{D}(T)$ reflects regularity and the $\mathscr{J}$-order on objects. Then the map $\bar{G}$ is an isomorphism from $\mathfrak{D}_{\ell}(S)$ to $\mathfrak{D}_{\ell}(T)$.

Proof. Let $H$ be a quasi-inverse of $G$. Then $\bar{H}$ and $\bar{G}$ are functional inverses of each other. Moreover, $\bar{G}$ and $\bar{H}$ are morphisms of preordered sets because $G$ reflects the $\mathscr{J}$-order. Since equivalences preserve the automorphism groups of objects, from Lemma 3.7 and the hypothesis that $G$ reflects regularity, we conclude that $\bar{G}$ preserves labels.

Proof of Theorem 6.3. Without loss of generality, we may assume that $S$ and $T$ have local units. Theorem 4.5 guarantees the existence of an equivalence $\mathbb{D}(S) \rightarrow \mathbb{D}(T)$ that reflects regularity and the $\mathscr{J}$-order on objects, whence Theorem 6.3 follows immediately from Lemma 6.4 
6.2.2. The rank of a $\mathscr{D}$-class is preserved under equivalences. Suppose we have a (right) action of a semigroup $S$ on a set $Q$. Then each element of $S$ can be viewed as a transformation on $Q$. Recall that the rank of a transformation is the cardinality of its image, and that $\mathscr{D}$-equivalent elements of $S$ have the same rank as transformations of $Q$. We modify the labeled preordered set $\mathfrak{D}_{\ell}(S)$ as follows: for each $\mathscr{D}$-class $D$ of $S$, instead of the label $(\varepsilon, H)$, consider the label $(\varepsilon, H, r)$, where $r$ is the rank in $Q$ of an element of $D$, viewed as an element of the transformation semigroup of $Q$ defined by the action of $S$ on $Q$. Denote by $\mathfrak{D}_{Q}(S)$ the resulting labeled poset.

Theorem 6.5. Let $S$ and $T$ be semigroups. Suppose that $S$ acts on the set $Q$, and $T$ acts on the set $R$. If $\mathbb{A}_{Q}$ and $\mathbb{A}_{R}$ are equivalent actions, then $\mathfrak{D}_{Q}(L U(S))$ and $\mathfrak{D}_{R}(L U(T))$ are isomorphic labeled preordered sets. Thus $Q \mapsto \mathfrak{D}_{Q}(L U(S))$ is a Karoubi invariant.

Proof. Replacing $S$ by $L U(S)$ and $T$ by $L U(T)$, we may assume without loss of generality that $S$ and $T$ have local units. By Corollary [5.6, there is an equivalence $G: \mathbb{D}(S) \rightarrow \mathbb{D}(T)$ such that $G$ reflects regularity and the $\mathscr{J}$-order on objects, and such that $\mathbb{B}_{Q}$ and $\mathbb{B}_{R} \circ G$ are equivalent functors. By Lemma 6.4, the map $\bar{G}: \mathfrak{D}_{\ell}(S) \rightarrow \mathfrak{D}_{\ell}(T)$ is an isomorphism of labeled preordered sets. Therefore, to prove that $\mathfrak{D}_{Q}(S)$ and $\mathfrak{D}_{R}(T)$ are isomorphic labeled preordered sets, it remains to show that $s$ and $G(s)$ have the same rank, for every $s \in S$. But the rank of $s$ in $Q$ is the cardinality of $\mathbb{B}_{Q}(s)$, and the rank of $G(s)$ in $R$ is the cardinality of $\mathbb{B}_{R}(G(s))$. Therefore, what we want to show follows from $\mathbb{B}_{Q}$ and $\mathbb{B}_{R} \circ G$ being equivalent functors.

\section{REFERENCES}

[1] F. Borceux. Handbook of categorical algebra. 1, volume 50 of Encyclopedia of Mathematics and its Applications. Cambridge University Press, Cambridge, 1994. Basic category theory.

[2] F. Borceux. Handbook of categorical algebra. 2, volume 51 of Encyclopedia of Mathematics and its Applications. Cambridge University Press, Cambridge, 1994. Categories and structures.

[3] F. Borceux. Handbook of categorical algebra. 3, volume 52 of Encyclopedia of Mathematics and its Applications. Cambridge University Press, Cambridge, 1994. Categories of sheaves.

[4] A. H. Clifford and G. B. Preston. The algebraic theory of semigroups. Vol. I. Mathematical Surveys, No. 7. American Mathematical Society, Providence, R.I., 1961.

[5] A. Costa. Conjugacy invariants of subshifts: an approach from profinite semigroup theory. Int. J. Algebra Comput., 16(4):629-655, 2006.

[6] A. Costa and B. Steinberg. A categorical invariant of flow equivalence of shifts. Ergodic Theory Dynam. Systems, to appear.

[7] V. Diekert and P. Gastin. Pure future local temporal logics are expressively complete for Mazurkiewicz traces. Inform. and Comput., 204(11):1597-1619, 2006.

[8] V. Diekert, M. Kufleitner, K. Reinhardt, and T. Walter. Regular languages are Church-Rosser congruential. In Automata, languages, and programming. Part II, volume 7392 of Lecture Notes in Comput. Sci., pages 177-188. Springer, Heidelberg, 2012. 
[9] V. Diekert, M. Kufleitner, and B. Steinberg. The Krohn-Rhodes theorem and local divisors. Fundamenta Informaticae, 116(1-4):65-7, 2012.

[10] S. Eilenberg. Automata, languages, and machines. Vol. B. Academic Press, New York, 1976. With two chapters ("Depth decomposition theorem" and "Complexity of semigroups and morphisms") by Bret Tilson, Pure and Applied Mathematics, Vol. 59.

[11] J. Funk, M. V. Lawson, and B. Steinberg. Characterizations of Morita equivalent inverse semigroups. J. Pure Appl. Algebra, 215(9):2262-2279, 2011.

[12] M. V. Lawson. Morita equivalence of semigroups with local units. J. Pure Appl. Algebra, 215(4):455-470, 2011.

[13] J. Leech. The $\mathscr{D}$-category of a semigroup. Semigroup Forum, 11:283-296, 1975/1976.

[14] D. Lind and B. Marcus. An introduction to symbolic dynamics and coding. Cambridge University Press, Cambridge, 1995.

[15] M. Loganathan. Cohomology of inverse semigroups. J. Algebra, 70(2):375-393, 1981.

[16] S. Mac Lane. Categories for the working mathematician, volume 5 of Graduate Texts in Mathematics. Springer-Verlag, New York, second edition, 1998.

[17] S. Mac Lane and I. Moerdijk. Sheaves in geometry and logic. Universitext. SpringerVerlag, New York, 1994. A first introduction to topos theory, Corrected reprint of the 1992 edition.

[18] J. Rhodes and B. Steinberg. The q-theory of finite semigroups. Springer Monographs in Mathematics. Springer, New York, 2009.

[19] B. Steinberg and B. Tilson. Categories as algebra. II. Internat. J. Algebra Comput., 13(6):627-703, 2003.

[20] H. Straubing. Finite semigroup varieties of the form V*D. J. Pure Appl. Algebra, 36(1):53-94, 1985.

[21] S. Talwar. Morita equivalence for semigroups. J. Austral. Math. Soc. Ser. A, 59(1):81111, 1995.

[22] B. Tilson. Categories as algebra: an essential ingredient in the theory of monoids. $J$. Pure Appl. Algebra, 48(1-2):83-198, 1987.

CMUC, Department of Mathematics, University of Coimbra, 3001-501 CoimBra, Portugal.

E-mail address: amgc@mat.uc.pt

Department of Mathematics, City College of New York, NAC 8/133, ConVent Ave at 138th Street, New York, NY 10031

E-mail address: bsteinberg@ccny.cuny.edu 